Crescendo, and Gilead, Speakers bureau: Sanofi, Horizon, Crescendo, Novartis, Genentech, Janssen, and AbbVie, Nasser Khan Shareholder of: AbbVie Inc., Employee of: AbbVie Inc., Jose Jeffrey Enejosa Shareholder of: AbbVie, Employee of: AbbVie, Yanna Song Shareholder of: AbbVie Inc., Employee of: AbbVie Inc., Jessica Suboticki Shareholder of: AbbVie Inc., Employee of: AbbVie Inc., Jeffrey R. Curtis Grant/research support from: Abbvie, Amgen, BMS, Corrona, Crescendo, Janssen, Pfizer, Regeneron/Sanofi, and UCB, Consultant of AbbVie, Amgen, BMS, Corrona, Crescendo, Janssen, Pfizer, Sanofi/Regeneron, and UCB

DOI: 10.1136/annrheumdis-2020-eular.2744

\section{THU0219 FIRST-INHUMAN STUDY OF SAFETY, PHARMACOKINETICS AND PHARMACODYNAMICS OF IRAK1/4 INHIBITOR R835 IN HEALTHY SUBJECTS}

L. Yan ${ }^{1}$, S. Tong ${ }^{1}$, A. Absalom², I. D. Daas ${ }^{3}$, G. Park ${ }^{1}$, V. Taylor ${ }^{1}$, D. Chow ${ }^{1}$ M. Lee ${ }^{1}$, H. Zheng ${ }^{1}$, A. Chow ${ }^{1}{ }^{1}{ }^{1}$ Rigel Pharmaceuticals Inc, South San Francisco, United States of America; ${ }^{2}$ University Medical Center Groningen, Groningen, Netherlands; ${ }^{3}$ QPS Netherlands BV, Groningen, Netherlands

Background: Toll-Like Receptors (TLR) and Interleukin-1 Receptors (IL-1R) play a critical role in the innate immune response as microbial and tissue damage sensors, providing a bridge between the innate and adaptive immunity. Interleukin receptor associated kinases (IRAK) 1 and 4 are serine/threonine kinases that are essential for signaling downstream of most TLRs and IL-1Rs and the resulting production of pro-inflammatory cytokines. Suppression of TLR and IL-1R signaling through inhibition of IRAK1/4 kinases is a promising therapeutic approach for the treatment of inflammatory and autoimmune diseases. We have identified a potent and selective IRAK1/4 inhibitor (R835) that showed dose-dependent inhibition of lipopolysaccharide (LPS, a TLR4 agonist), and $\mathrm{IL}-1 \beta$ induced serum cytokines in mice. R835 prevented disease onset and progression in multiple rodent models of inflammatory diseases, including arthritis and lupus models.

Objectives: The aim of this FIH study was to characterize the safety, pharmacokinetics (PK) and pharmacodynamics (PD) of R835 after single or multiple dose oral administrations.

Methods: This study was a randomized, placebo-controlled, double-blind Phase 1 study in healthy subjects in three parts: single ascending doses $(20 \mathrm{mg}-1920 \mathrm{mg}$ Part A) with food effect in a separate cohort $(480 \mathrm{mg})$, multiple ascending doses (120 mg and $960 \mathrm{mg}, \mathrm{BID}$, Part B) with a caffeine interaction (960 mg cohort), and an intravenous LPS challenge test at $240 \mathrm{mg}$ oral dose of R835 (Part C).

Results: Single doses of up to $480 \mathrm{mg}$ R835 in organic solution, single doses of up to $1920 \mathrm{mg}$ R835 as capsule, multiple doses of $120 \mathrm{mg}$ R835 $\mathrm{Q} 12 \mathrm{H}$ (organic solution), and $960 \mathrm{mg} \mathrm{R} 835 \mathrm{Q} 12 \mathrm{H}$ (capsule) were safe and well tolerated. All R835 related adverse events (AEs) were mild in intensity and reversible, and mostly associated with the higher doses of R835 in the organic solution. The most common AEs were headache and gastrointestinal disturbance. The PK of R835 was linear and dose proportional in exposure over the dose range studied. A nominal level of accumulation in plasma achieved rapidly upon repeated BID administrations with steady-state essentially attained in 2 days. A high-fat meal with the capsule formulation resulted in slow rate of absorption but had no effect on the extent of absorption. There was no effect of R835 on metabolism of caffeine (P450 CYP1A2 prototype substrate). In the LPS challenge test, R835 profoundly inhibited the acute release of cytokines, including TNF-a, IL-6, IL-8, MIP1 $a$ and MIP1 $\beta$, but had no impact on CRP release.

Conclusion: R835 was well tolerated after single or multiple dose administrations. The most common AEs were headache and gastrointestinal disturbance. For both of the formulations tested, the PK of R835 was linear and exposure was dose proportional with rapid steady-state attainment following BID administration. There was no drug-drug interaction by use of caffeine as the protype substrate. R835 inhibited the LPS induced release of cytokines in the serum, including TNF-a, IL-6, IL-8, MIP1 $a$ and MIP1 $\beta$, mirroring preclinical data in mice. The desirable $\mathrm{PK}$ and safety profile combined with proof of mechanism, as demonstrated by inhibition of cytokine release, support progression of R835 into Phase II clinical development as an agent for the treatment of inflammatory and autoimmune diseases.

Disclosure of Interests: : Lucy Yan Shareholder of: Amgen, Rigel, Employee of: Amgen, Rigel, Sandra Tong Shareholder of: Rigel, Employee of: Rigel, Anthony Absalom: None declared, Izaak den Daas: None declared, Gary Park Shareholder of: Rigel Pharmaceuticals, Employee of: Rigel Pharmaceuticals, Vanessa Taylor Shareholder of: Rigel Pharmaceuticals, Employee of: Rigel Pharmaceuticals, Donna Chow Shareholder of: Rigel, Employee of Rigel, Meng Lee Shareholder of: Rigel, Employee of: Rigel, Hanzhe Zheng Shareholder of: Rigel, Employee of: Rigel, Andrew Chow Shareholder of: Rigel, Employee of: Rigel

DOI: 10.1136/annrheumdis-2020-eular.4590
THURSDAY, 04 JUNE 2020

\section{SLE, Sjögren's and APS - etiology, pathogenesis and animal models}

\section{THU0220 AUTOPHAGY IN SJOGREN'S SYNDROME SALIVARY GLAND EPITHELIAL CELLS (SGECS) IS ASSOCIATED WITH THE SEVERITY OF INFLAMMATION AND EPITHELIAL CELLS ACTIVATION.}

S. Colafrancesco ${ }^{1}$, C. Barbati ${ }^{1}$, V. Iannizzotto ${ }^{2}$, L. Mastromanno ${ }^{1}$, S. Nayar ${ }^{2}$, E. Pipi ${ }^{2}$, A. Gattamelata ${ }^{1}$, F. Ciccia ${ }^{3}$, C. Alessandri ${ }^{1}$, F. Barone ${ }^{2}$, F. Conti ${ }^{1}$, R. Priori ${ }^{1} .{ }^{1}$ Sapienza University of Rome, Dipartimento di Scienze Cliniche Internistiche Anestesiologiche e Cardiovascolari, Rome, Italy; ${ }^{2}$ University of Birmingham, Institute of Inflammation and Ageing, Birmingham, United Kingdom; ${ }^{3}$ Università Degli Studi Della Campania L. Vanvitelli, Napoli, Italy

Background: Sjögren's Syndrome (SS) is characterized by chronic inflammation supported by intrinsic activation of salivary gland epithelial cells (SGECs). Eventually, apoptosis of SGECs ensues, which leads to salivary gland dysfunction and exposition of autoantigens. Autophagy is a stress coping mechanisms of cells implicated in both survival and exposition of autoantigens, and is thereby plausibly implicated in the pathogenesis of SS. At present, the exact relationship between apoptosis and autophagy in SS SGECs is unclear, as is the link between these mechanisms and SGECs activation.

Objectives: To explore autophagy in SGECs from patients with SS and to evaluate its relationship with apoptosis and SGECs activation.

Methods: Consecutive patients with suspected SS referring to our "Sjogren Clinic" were enrolled, and minor salivary gland (MSG) biopsies were collected for: (1) SGECs culture, (2) PCR analysis, (3) IFI analysis. In SGECs cultures, the expression of autophagy (LC3II), apoptosis (annexin V/PI) and adhesion molecules (ICAM) was investigated by flow cytometry (results expressed as mean $\% \pm \mathrm{SD}$ ). The expression of the autophagy gene MAP1LC3II was evaluated by PCR (expressed as $2^{\wedge}$ deltaCT normalized to GADPH) on both MSG sections and MSG acinar and ductal epithelium samples obtained by laser capture microdissection. Tissue expression of LC3II was evaluated by IFI on SS MSG.

Results: Primary SGECs cultures were established from 14 MSG obtained for diagnostic purposes (SS $n=8$, Sicca $n=6$ ). These cells exhibited an inverse correlation between apoptosis and autophagy $(\mathrm{p}=0.007, \mathrm{r}=-0.784)$, with lower levels of apoptosis $(19.7 \pm 6.5$ vs $24.5 \pm 8.5, p=n s)$ and higher levels of autophagy $(59.7 \pm 13.1$ vs $54.19 \pm 19.4, p=n s)$ in SS compared to Sicca. In SS, MAP1LC3 was positively cor related with Focus Score $(\mathrm{p}=0.021 \mathrm{r}=0.478)$; however, PCR studies did not reveal significant differences in MAP1LC3 expression between SS $(n=26)$ and Sicca $(n=15)(0.024 \pm 0.010$ vs $0.022 \pm 0.008, p=n s)$. Ductal SGECs $(n=4)$ isolated by laser microdissection of MSG revealed a higher expression of MAP1LC3 $(0.005 \pm 0.0005$ vs $0.003 \pm 0.0008 ; p=0.057)$ compared to normal acinar epithelium $(n=5)$; a major expression of LC3II in ducts was confirmed by IFI (Image).

In SS, a higher expression of ICAM compared to sicca was observed $(11.1 \pm 3.8$ vs $6.9 \pm 6.9, p=0.006)$ and autophagy and apoptosis showed a trend of positive and negative correlation with this molecule, respectively $(p=0.683 r=0.118$ and $\mathrm{p}=0.106 \mathrm{r}=-0.446)$.

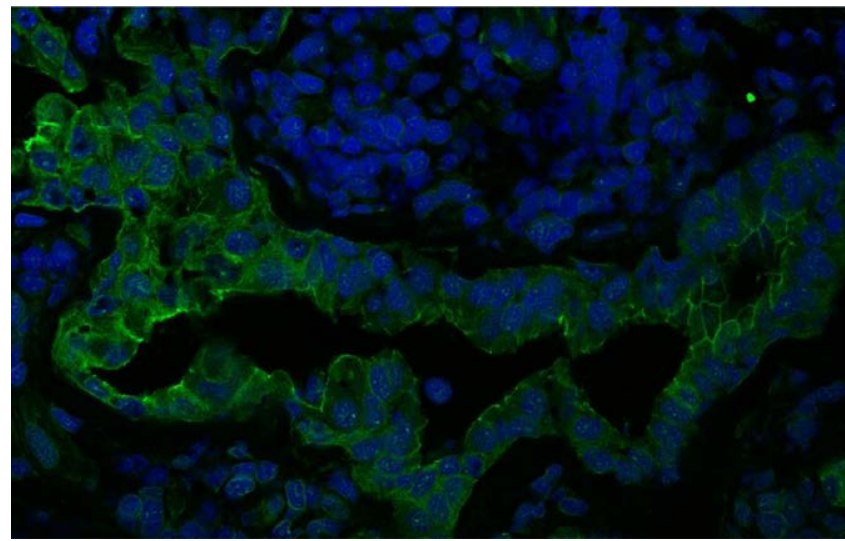

Figure. LC3-II staining in SS MSG [LC3-II+ (green) and Hoechst stain (b/ue); 60x magnification].

Conclusion: In SS, autophagy is upregulated in SGECs and inversely correlated with apoptosis, thus supporting a role of this process in cells' death prevention during inflammatory process. Indeed, the degree of msg inflammation is correlated more with the activation of autophagy than apoptosis. Interesting, in SS, SGECs autophagy is mainly observed at ductal level and is correlated with 
higher expression of adhesion molecules suggesting a link between this pathway and changes in SGECs immune phenotype.

Disclosure of Interests: : Serena Colafrancesco: None declared, cristiana barbati: None declared, Valentina lannizzotto: None declared, Linda Mastromanno: None declared, Saba Nayar: None declared, Elena Pipi: None declared, angelica gattamelata: None declared, francesco ciccia Grant/research support from: pfizer, novartis, roche, Consultant of: pfizer, novartis, lilly, abbvie, Speakers bureau: pfizer, novartis, lilly, abbvie, cristiano alessandri Grant/research support from: Pfizer, Francesca Barone: None declared, fabrizio conti Speakers bureau: BMS, Lilly, Abbvie, Pfizer, Sanofi, Roberta Priori: None declared DOI: 10.1136/annrheumdis-2020-eular.5212

\begin{tabular}{|l|l}
\hline THU0221 & EVIDENCE FOR A PATHOGENIC ROLE OF EXTRA- \\
& FOLLICULAR, IL-10 PRODUCING CCR6+B-HELPER \\
& T-CELLS IN SYSTEMIC LUPUS ERYTHEMATOSUS
\end{tabular}

M. Gerosa ${ }^{1,2}$, F. Facciotti ${ }^{3}$, P. Larghi ${ }^{3}$, R. Bosotti ${ }^{3}$, C. Vasco $^{3}$, N. Gagliani ${ }^{4,5}$, C. Cordiglieri ${ }^{3}$, E. Rottoli ${ }^{6}$, A. E. Penatti ${ }^{7}$, L. M. Argolini ${ }^{2}$, B. Karnani ${ }^{3}$,

Y. Kobayashi ${ }^{4}$, M. Bombaci ${ }^{3}$, J. P. Van Hamburg ${ }^{8}$, R. Gualtierotti ${ }^{9}$, S. Gatti ${ }^{10}$

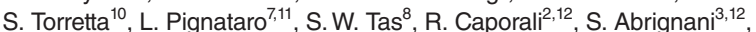
M. Pagani ${ }^{3,9}$, F. Grassi ${ }^{3,6,9}$, P. L. Meroni ${ }^{13}$, R. Flavell ${ }^{4}$, J. Geginat ${ }^{3} .{ }^{1}$ University of Milan, Department of Clinical Science and Community Health, Milan, Italy; ${ }^{2}$ ASST PINI-CTO, Clinical Rheumatology Unit, Lupus Clinic, Milan, Italy; ${ }^{3}$ INGMNational Institute of Molecular Genetics “Romeo ed Enrica Invernizzi”, Milan, Italy; ${ }^{4}$ Department of Immunobiology, School of Medicine, Yale University, New Heaven, United States of America; ${ }^{5}$ Department of Medicine \& Department of General, Visceral and Thoracic Surgery, University Medical Center Hamburg-Eppendorf, Hamburg, Germany; ${ }^{6}$ Institute for Research in Biomedicine, Bellinzona, Switzerland; ${ }^{7}$ University of Milan, Milan, Italy; ${ }^{8}$ Amsterdam UMC, University of Amsterdam, Academic Medical Center, Amsterdam, Netherlands; ${ }^{9}$ Department of Medical Biotechnology and Translational Medicine, University of Milan, Milan, Italy; ${ }^{10}$ Fondazione IRCCS Cá Granda, Ospedale Maggiore Policlinico, Milan, Italy; ${ }^{11}$ Fondazione IRCCS Cá Granda, Ospedale Maggiore Policlinico Milano, Milan, Italy; ${ }^{12}$ Department of Clinical Science and Community Health, University of Milan, Milan, Italy; ${ }^{13}$ IRCCS Istituto Auxologico Italiano, Milan, Italy

Background: IL-10 plays a key role in systemic lupus erythematosus (SLE) pathogenesis, promoting B-cell response. IL10 is mainly secreted by regulatory T-cells, but follicular helper T-cells $\left(T_{F H}\right)$, also produce it. We previously identified a subset of CCR6 ${ }^{+} \mathrm{IL}-7 \mathrm{R}^{+} \mathrm{T}$-cells in human tonsils providing IL-10-dependent $\mathrm{B}$-cell help. These $\mathrm{CCR} 6^{+} \mathrm{T}$-cells were able to produce IL-10, inducing IgG production. Objectives: to investigate a possible role of $\mathrm{CD}^{+} \mathrm{CCR} 6^{+} \mathrm{IL} 7 \mathrm{R}^{+} \mathrm{T}$-cells in SLE pathogenesis.

Methods: 37 patients fulfilling the ACR criteria for SLE have been included. Disease activity was assessed by $2 \mathrm{k}$-SLEDAI. PBMC were analyzed by flow cytometry, using specific lineage markers. CCR6 ${ }^{+} I L 7 R^{+} T$-cells purified from total PBMC of SLE patients or healthy donors (HD) were co-cultured with autologous CD20 ${ }^{+}$B-cells. IL-10, II-17, total IgG and anti-dsDNA antibodies titers in patients serum and culture supernatants were assessed by ELISA. Embedded sections of lymph nodes from 8 SLE patients were analyzed by immunofluorescence (IF). Results: IL10 levels were significantly higher in SLE patients (Fig 1A). CD4 ${ }^{+} \mathrm{C}-$ $\mathrm{CR6}^{+}$IL7R ${ }^{+} \mathrm{T}$-cells were significantly increased in SLE, in particular in those with higher disease activity and higher IL10 levels. CD4 ${ }^{+} C C R 6^{+} I L 7 R^{+} T$-cells levels associated with anti-dsDNA positivity. CCR6 ${ }^{+} I L 7 R^{+} T$-cells of SLE patients induced production of IgG and anti-dsDNA IgG (in anti-dsDNA + patients) from autologous $\mathrm{B}$-cells, providing spontaneous help for autoantibody production ex vivo (Fig 1BC). The IF study of lymph nodes of SLE patients showed that IL-10-producing CCR6 ${ }^{+}$T-cells were highly abundant and co-localized with B-cells at follicle margins.

A)

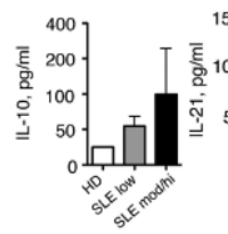

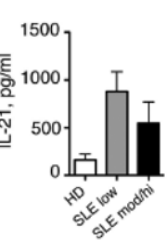

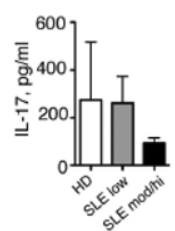

B)

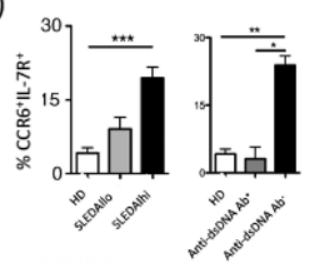

C)

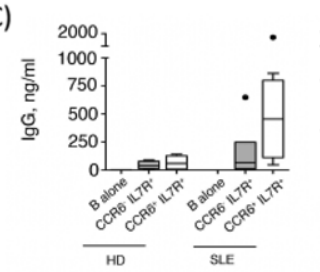

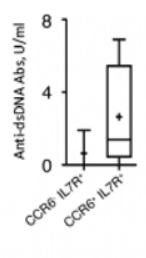

Conclusion: our study revealed a novel population of extra-follicular B-helper T-cells, which produce IL-10 and could play a prominent pathogenic role in SLE. Further studies will clarify if this potentially pathogenic cell population might represent a possible future therapeutic target.

References:

[1] Facciotti F. J Allergy Clin Immunol. 2016; Geginat J. Semin Immunol. 2019; Tsokos GC. Nat Rev Rheumatol. 2019

Tab 1: SLE patients characteristics

\begin{tabular}{lc}
\hline & $(\mathrm{n}=37)$ \\
Demographics & $37 / 5$ \\
Female/Male, $\mathrm{n}$ & $44(38-49)$ \\
Age, years, median (IQR) & $19(11-26)$ \\
Disease duration, years, median (IQR) & \\
Lab tests & $86 \%$ \\
ANA & $46 \%$ \\
*anti-dsDNA (\%) & $41 \%$ \\
$\quad$ medium/high titre & \\
Disease activity and clinical manifestations & $3.5(0-24)$ \\
$\quad$ SLEDAl-2K, median (min-max) & $19 \%$ \\
$\quad$ Moderate/high activity & \\
Ongoing therapy & $7,5 \mathrm{mg}(2,5-20)$ \\
Prednisone dose mg/day, median (IQR) & $78 \%$ \\
hydroxychloroquine & $87 \%$ \\
Immunosuppressants & \\
\hline
\end{tabular}
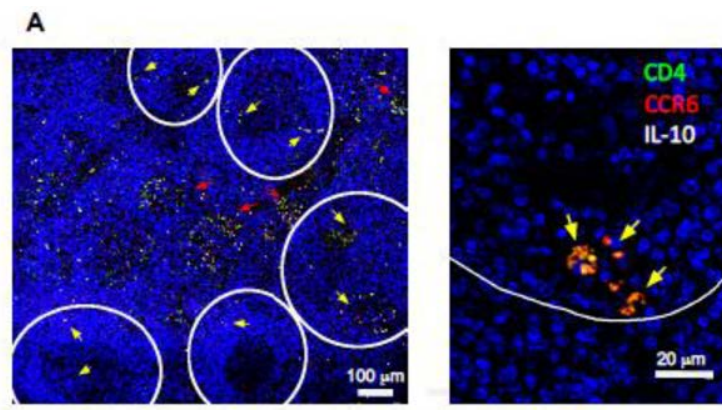

B

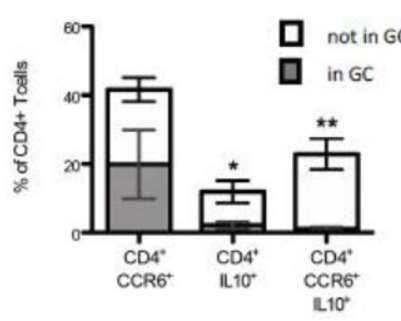

C
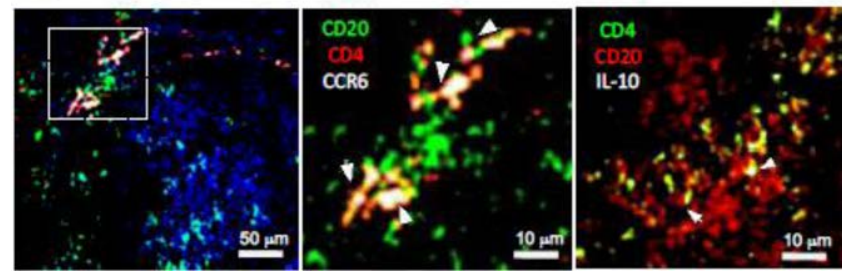

Fig 2

Disclosure of Interests: : Maria Gerosa: None declared, Federica Facciotti None declared, Paola Larghi: None declared, Roberto Bosotti: None declared, Chiara Vasco: None declared, Nicola Gagliani: None declared, Chiara Cordiglieri: None declared, Elsa Rottoli: None declared, Alessandra Emiliana Penatti: None declared, Lorenza Maria Argolini: None declared, Bhavna Karnani: None declared, Yasushi Kobayashi: None declared, Mauro Bombaci: None declared, Jan Piet Van Hamburg: None declared, Roberta Gualtierotti: None declared, Stefano Gatti: None declared, Sara Torretta: None declared, Lorenzo Pignataro: None declared, Sander W. Tas: None declared, Roberto Caporali Consultant of: AbbVie; Gilead Sciences, Inc.; Lilly; Merck Sharp \& Dohme; Celgene; Bristol-Myers Squibb; Pfizer; UCB, Speakers bureau: Abbvie; Bristol-Myers Squibb; Celgene; Lilly; Gilead Sciences, Inc; MSD; Pfizer; Roche; UCB, Sergio Abrignani: None declared, Massimiliano Pagani: None declared, Fabio Grassi: None declared, Pier Luigi Meroni: None declared, Richard Flavell: None declared, Jens Geginat: None declared DOI: 10.1136/annrheumdis-2020-eular.1532 